\title{
ANÁLISS ECONÓMICO DEL COSTO DE VIDA EN LA FRONTERA NORTE DE COAHUILA
}

\begin{abstract}
RESU MEN
El objetivo de este trabajo es realizar una reflexión acerca del cambio de estrategia en la determinación del salario y la ruptura de su relación con la canasta básica y el poder adquisitivo de los trabajadores. Pretendemos evidenciar el caso particular de las ciudades en la frontera norte de Coahuila. Bajo la nueva estrategia de desarrollo en el ámbito de los salarios, dada la pérdida de relación de éstecon el costo devida de la población y sus necesidades básicas, el logro de los objetivos de equilibrio macroeconómico ha sido a través de la contención salarial; sin embargo, esta estrategia no ha traído otro resultado más que la mayor pérdida de bienestar de la población. Los cambios habidos en la política salarial, han mermado la capacidad de gran parte de la población de acceder a los elementos necesarios de satisfacción básica, situación que se agrava más considerando las diferencias en el costo económico de vivir en determinadas ciudades, concretamente en ciudades fronterizas.
\end{abstract}

\section{A BSTRACT}

The purpose of this work is to reflect on the change of strategy that was taken with regards to the occurrence of a rupture of the relation between the salary and the basic household supply and purchasing power of the workers. Weare particularly interested in evidencing the case of the cities al ong the N orthern border of Coahuila. In view of the loss of the relationship between the salary and the cost of life of the population and its basic needs, under the new development salary strategy, the attainment of the macroeconomic equilibrium aims have been by means of a salary restraint. N onetheless, this strategy has brought about no other outcome but the greatest loss of well-being among the population. The changes that havetaken place in the salary policy have undermined the capabilities of most of the population in having access to the elements deemed necessary for a scheme of basic satisfaction. Such scenario is agravated by the differences existing in the economic cost of living in certain cities, mainly along border cities.

\footnotetext{
* Investigadora de El Colegio de la frontera norte, Dirección Regional de nuevo laredo, TAMAULIPAS. Correo electrónico: bvazquez@colef.mx
} 


\section{INTRODUCCIÓN}

En los últimos años, la pérdida de poder adquisitivo del salario real ha sido muy profunda, y se ha dado como resultado del encarecimiento de los elementos de consumo diario de la población, es decir, en su costo de vida económico. La caída del salario real ha estado enmarcada por sucesos económicosquesehan presentado en México desdefinales delos años setenta, crisis recurrentes, aplicación deprogramas deajuste y choque estructural, entre otros factores. En los últimos veinte años, la estrategia del modelo económico sobre el salario ha variado en su dinámica, pues se han presentado cambios importantes en la política salarial, lo que para algunos autores representa un alejamiento del Estado en la determinación, precisamente, de la política sal arial, y ha conducido hacia una posición más flexible y menos comprometida por parte del mismo (Marinakis, 1995; Solis de Alba, 1995).

Lo cierto es que para el actual periodo de gobierno (1999), y el que le precedió, la política salarial ha sido una de las estrategias más importante para alcanzar el equilibrio macroeconómico tan buscado en nuestro país. En tal contexto, la intervención del Estado en el mercado de trabajo se ha dado a través de instituciones, de mecanismos e instrumentos, pues con ellos se han logrado fijar montos de salario que no ponen en riesgo el equilibrio del modelo. Particularmente esta intervención fueimportantedurantela crisis económica de los ochenta, puesse requirió del establecimiento detopes salariales quecontrolaran los niveles inflacionarios al canzados, utilizando para ello la cultura de los pactos económicos entre los sectores productivos. Así, restablecer el equilibrio macroeconómico seconvirtió en el objetivo prioritario que condicionó a los objetivos de política económica, inclusive sociales y dedistribución del ingreso.

Mástarde, la función del Estado - en esteterreno- selimitaría la simple fijación de normas generales y sectoriales para los reajustes sal ariales. En al gunos casos, estos reajustes siguieron en forma directa las variadas políticas económicas (Marinakis, 1995); de este modo, la contención salarial establecida por las autoridades, seconstituyó en la premisa básica del sector público y en pauta a seguir para el sector privado. $^{1}$ 
El objetivo deestetrabajo es real izar una reflexión acerca del cambio deestrategia en la determinación del salario y la ruptura desu relación con el costo de vida de la población y con su referente económico inmediato: la canasta básica y el poder adquisitivo de los trabajadores. Pretendemos evidenciar el caso particular delas ciudades en la frontera norte de Coahuila.

DEL SALARIO MÍNIMO A LA FLEXIBILIZACIÓN SALARIAL

Originalmente la fijación del salario mínimo (SM), de acuerdo con la Constitución de1917 (fracción IX del artículo 123), asegura al trabajador un valor de su fuerza de trabajo tal que le permita la reproducción física y social propia y desu familia. En estesentido, lecorrespondeal Estado mexicano vigilar y establecer instrumentos, mecanismos e instituciones que, basados en la Ley federal del trabajo (LFT), atiendan esta situación y concilien o resuel van los intereses contrapuestos.

A partir de 1931 la fijación del SM se da a través de las comisiones especiales formadas en cada municipio, mismas que estaban subordinadas a la Junta Central de Conciliación en cada estado. La primera modificación a la LFT vino a reforzar el sistema defijación de SM por comisiones especiales integradas en cada municipio. ${ }^{2}$

A lo largo del proceso de industrialización por sustitución de importaciones (ISI), y aun en el proceso de conformación del Estado benefactor, en lo referentea la determinación sal arial sedecidió quela fijación delos SM debía ser por zonas económicasy no por municipios,

${ }^{1}$ La participación del Estado en la fijación desalarios creció con su poder dearbitraje en las negociaciones colectivas. Posteriormente, su participación se delimitó a un papel de mediador entre los factores dela producción; para un mayor tratamiento véase: Andrés Marinakis, (1995).

2 “[...]derivadas principalmente, de que la división municipal, producto de diversos fenómenos históricos y accidentes geográficos, no guardaba relación alguna con las características de desarrollo económico regional y con otros fenómenos económicos de alcance más general, por lo que no podría servir de fundamento para la determinación de los SM en condiciones adecuadas" (STyps, 1997: http:/ / stps.gob.mx). 
de tal manera que las reformas a la fracción VI del artículo 123 constitucional y las correspondientes a la LFT en materia de salario mínimo, en 1963originaron la creación de una comisión nacional y 111 comisiones regionales de los SM en el país, sistema que permaneció hasta 1986. Posteriormente, en eseaño (1986), el Congreso dela Unión aprobó una iniciativa del ejecutivo para reformar el esquema, el cual plantea que a partir del 1 de enero de 1987 sea la Comisión N acional deSalarios Mínimos (CNSM), integrada por representantes del gobierno, sector empresarial y laboral, la que dictelos montos salariales mínimos de pago en el país, desintegrándose el Sistema de Comisiones Regionales vigente hasta el 31 de diciembre de 1986 (DOF-23/ 12/ 86).

Con este nuevo esquema se pretende una mayor flexibilidad territorial en la fijación de los SM, pues éstos serán fijados por áreas geográficas integradas por uno o más municipios, de una o más entidades federativas, lo que permitiría corregir inconsistencias observadas en cuanto a las zonas de aplicación de los SM, así como considerar las características particulares de las áreas geográficas de rápido desarrollo o con características especiales.

Sin embargo, estehecho marca también el comienzo de una nueva modalidad de revisión y acuerdos salariales, una modalidad de imposición gubernamental, deacuerdosy aceptación total deun nuevo esquema de crecimiento y desarrollo económico nacional, donde la nueva política salarial se vuelve facilitadora de la productividad, concepto eje de las nuevas relaciones entre capital y trabajo; pese a una retórica de oposición y desacuerdo de dirigentes sindicales en la búsqueda de mejoras sal ariales para sus agremiados.

Hasta finales de los años setenta presenciamos el establecimiento deinstituciones y medidas que protegían la capacidad adquisitiva del salario y facilitaban el acceso de los trabajadores a la obtención de los satisfactores básicos. En 1971, el gobierno mexicano firmó un convenio establecido por la Organización Internacional del Trabajo (OIT), ${ }^{3}$

${ }^{3}$ La OIT, creada en 1919y asociada a la oNU desde 1947, tienecomo objetivo promover la justicia social con el mejoramiento delas condiciones devida y detrabajo. Desde su fundación, este organismo se ha abocado a la tarea de elaborar normas 
referente a la fijación de cantidades mínimas de salario para los trabajadores; en esteconvenio seestablecen los elementos a considerar para su determinación: las necesidades de los trabajadores y sus familias, el costo de vida y sus variaciones; los factores económicos, incluidos los requerimientos del desarrollo económico, los niveles de productividad, así como la conveniencia de alcanzar y mantener un alto nivel deempleo.

Serecomienda quesu fijación debe constituir un el emento de toda política establecida para el iminar la pobreza y asegurar la satisfacción de las necesidades de todos los trabajadores y sus familias, así como proporcionarles la protección social respecto delosmínimos permisibles desalarios. Sin embargo, no es hasta 1974 quesereconoceel derecho a la seguridad social detodo trabajador asalariado, y hasta 1978 cuando se establece el derecho al trabajo (Ordóñez, 1997).

Esta visión distributiva para mantener condiciones decorosas en el nivel devida dela población a través del establecimiento delosmontos salariales (considerada de utilidad pública), continuó hasta losochenta. En los primeros años de la crisis económica se pudo observar un retroceso en tales objetivos; en 1982, el SM aumentó 63.8\%, mientras quela inflación fue de $98.84 \%$; en 1985, las cifras fueron $48.6 \%$ contra $63.75 \%$, respectivamente, mientras que para 1987 , su comportamiento fue de $159.7 \%$ contra $106 \%$.

Posterior a la unificación de las zonas salariales en 1986, inició la tradición de los pactos y acuerdos en el establecimiento de montos sal arial es topeentretrabajadores, empresarios y gobierno, mismosque se prorrogarían una década completa; sin embargo, este periodo también marcaría el inicio de un modelo económico de apertura económica dondeel Estado deja detener funciones detipo económico para, en el ámbito laboral, dar paso a una política decontención salarial

laborales internacionales, detal forma que su trabajo ha influido y modificado las estructuras administrativas, económicas y sociales que se van dando en el mundo donde los trabajadores cumplen un papel importante. Sobrelo anterior, en 1970 se firmó el convenio número 131, referentea la fijación desalarios mínimos, queentró en vigor el 29 de abril de 1972, mismo que se ratificaría en 1981 por el gobierno de López Portillo. Véase: Oficina Internacional del Trabajo (OIT), 1992. 
no dependiente directamente de él, sino más en función directa de criterios de productividad y eficiencia económica; es decir, una política salarial en función dela disponibilidad delas empresas y del "poder" denegociación delos trabajadores.

\section{LA NUEVA POLÍTICA LABORAL Y SALARIAL}

La disminución dela participación delossalarios en el ingreso nacional a lo largo del periodo de ajuste pone en entredicho el objetivo de la gestión del desarrollo: la búsqueda deuna mejora continua en el nivel devidadela población. Esclaro quedesdemediados delosaños setenta se ha observado un estancamiento de los salarios reales, resultado de las crisis económicas y de una tendencia a la retirada del Estado desus funciones como Estado benefactor, situación que ha dado pie al establecimiento de un nuevo modelo dedesarrollo donde, en el ámbito laboral, seha tendido hacia la flexibilización delas relaciones sal ariales.

La nueva cultura laboral se basa fundamental mente en el acuerdo deremuneraciones al trabajo dado su nivel deproductividad, alejando al SM dela obligatoriedad y características dictadas por la C on stitución y por la LFT, y tomándolo simplemente como referencia en las negociaciones contractuales con grupos sindicales, eigualmentecomo referencia tope para el pago de otras prestaciones históricamente logradas, surgiendo, de esta manera, la parte variable de la remuneración al trabajo: el salario integral, mismo que bonifica productividad, fidelidad e incondicionalidad de los empleados a los empleadores a la hora de adecuarse a los requerimientos del mercado de trabajo, y donde, además, se deja al margen la injerencia sindical (Solís de Alba, 1995: 235).

Este nuevo enfoque en la determinación de los salarios -y por lo tanto de distribución del ingreso- menciona queel nivel de aquéllos se establecerá conforme el nivel de productividad del trabajo. Sin embargo, la evidenciaa lo largo delos últimosquinceaños ha mostrado que la asignación de las remuneraciones al trabajo dictadas por el mercado (o por quienes creen en su virtual y equitativo proceso de distribución) ha generado amplias situaciones de intolerancia en las condiciones socioeconómicas dela población, detal forma quesecreó 
una cadena de vicios que difícilmente generará condiciones hacia el aumento dela productividad y competitividad al nivel exigido por el mercado.

Por otra parte, aún no sehan encontrado los mecanismos económicos que aseguren, evalúen y cuantifiquen una relación directa entre productividad y salario, de tal forma que ante estos cambios los trabajadores han quedado desprotegidos en la determinación de la remuneración a su fuerza de trabajo y, en consecuencia, "Ia caída de los salarios reales provocó que las familias mexicanas, en defensa de sus condiciones devida, echaran mano detodos los medios asu alcance para contrabalancear la disminución de sus ingresos", básicamente aumentando los niveles de autoexplotación de los integrantes del hogar (Cortés y Rubal cava, 1991:10).

Finalmente, bajo estenuevo esquema en las relaciones laborales, se pretende olvidar y pasar de largo los criterios fundamental es para la fijación de los salarios en la Constitución y en la LFT; como si la productividad del trabajo fuesehomogénea entre los diversos sectores, ramas y actividades desarrolladas, y como si la competitividad en mercados gl obales unificad os implicara de facto la homogeneización de los satisfactores que cubren las necesidades esenciales de toda la población trabajadora.

\section{COSTO DE VIDA EN LA FRONTERA NORTE}

La caída constante del nivel real del salario y su poder adquisitivo, desdemediados dela década delos setenta, ha afectado negativamente en forma generalizada el nivel de consumo de la población. Sin embargo, esta situación ha mostrado efectos diferenciados en las diversas regiones del país; es decir, el resultado de la estrategia económica ha sido asimilado de manera distinta por las diversas estructuras económicas y grupos sociales; un claro ejemplo lo constituyen las ciudades de la frontera norte de México, las cuales, al mantener una constanteactividad económica y niveles deempleo más el evados al promedio nacional, han resentido en menor grado la caída del nivel de bienestar dela población, este último en el contexto dela situación que se presenta en otras regiones del país. 
En este sentido, la relación entre el costo de vida económico y un salario adecuado que garantice la reproducción física y social de una familia ha perdido todo vínculo. De hecho, es posible que en ningún periodo de tiempo esta relación se haya establecido de manera equitativa, pues de una sociedad a otra los estilos devida y los hábitos de consumo de la población difieren y, por lo tanto, el costo de los satisfactores necesarios diferirá igualmente. La dificultad estriba en cómo establecer satisfactores homogéneos de calidad de vida, y consecuentemente, un salario adecuado que cubra las necesidades esenciales de los trabajadores y sus familias.

El papel deEstado, en todo caso, desde 1917 y con la expedición de la LFT, era precisamente ser vigilantey garantizar lo establecido por la legislación, haciendo un intento de medir con cierta aproximación las necesidades mínimas de una familia típica y el costo que implica su satisfacción, de tal forma que el aumento o mejora continua del nivel de bienestar social de la población nacional diera los elementos que Ilevasen al aumento dela productividad del trabajo y con ello mayores ventajas de competitividad en los mercados.

El punto es cómo determinar las necesidades de los trabajadores, sustentadas en un nivel de vida socialmente aceptable, cómo transformar necesidades humanas en necesidades concretas y particulares en un ámbito social específico. Boltvinik menciona quelos elementos que fundamentan las necesi dades sociales concretas son: la naturaleza del proceso deproducción y delas actividades económicas; y la natural eza del proceso deconsumo, las condiciones derestitución y reproducción de la fuerza de trabajo.

De esta forma, las necesidades sociales son determinadas y normadas social mente, pues es la misma población la que, deacuerdo con el sistema de producción y consumo en el que se desenvuelve, adopta satisfactores, bienes y servicios para dichas necesidades, así como condiciones quelas satisfacen (Boltvinik J., 1995:5-6). Setrata de evaluar la realidad de cada país, región, sociedad, etcétera, en su contexto temporal. Los estilos devida semodifican en espacio y tiempo.

Resolver este problema requiere de un trabajo de investigación minucioso que aborde el estudio de estos elementos delimitando temporal y espacialmentesu realidad específica. Así pues, una encuesta 
a hogares levantada en 1997 para la ciudad fronteriza de Piedras Negras, Coahuila, mostró la contradicción entre la naturaleza del proceso de producción y consumo, ya quela estructura económica de esta localidad, y específicamente las remuneraciones al trabajo productivo, secontraponen con un estilo de vida fronterizo, un patrón deconsumo queimplica un elevado costo económico.

En esta investigación se determinó el costo del consumo alimenticio observado, no normativo, para una familia promedio de 4.45 miembros en unidades de adulto equivalente, es decir un hogar integrado por 2.82 adultos mayores de 15 años, 1.23 niños entre 4 y 14 años, y 0.38 bebés menores de 4 años. Se establecieron consumos per cápita por grupo alimenticio que, al contraponersecon el precio delos productos en el mercado, fue posibleobtener un costo diario de 30.96 pesos para cubrir los satisfactores al imenticios para la reproducción física en este contexto fronterizo (ver cuadro 1). A esta situación habría queagregar que no se trata de consumo normativo; es decir, en términos de los requerimientos nutricionales indispensables, sin embargo, esta referencia da idea delas implicaciones económicas para la satisfacción delas necesi dades al imenticias.

En este contexto, un análisis de las variaciones en precios en los mercados fronterizos muestra cómo la brecha existente entre el costo económico de vivir en la frontera (los índices de inflación local) y las remuneraciones a la fuerza de trabajo, se hace más profunda, a pesar de las relativas ventajas para la región, derivadas de su dinámica de crecimiento económico. Por ejemplo, del total nacional de empleos generados por la industria maquiladora, poco más del $8 \%$ se ubican en el estad o deCoahuila; deéste, más de $60 \%$ segenera en los municipios fronterizos de Piedras N egras y A cuña. Esta última ciudad, en 1993, ocupaba 18749 empleos maquiladores; para agosto de 1998, ya eran 31622 personas. Piedras Negras, por su parte, pasó de 9046 a 14 898, respectivamente. ${ }^{4} A$ nteesta situación, y en vista deque, a decir por las 
cifras oficiales, el repunte de las exportaciones no petroleras se basa fundamental mente en la industria manufacturera, en mayor medida la industria maquiladora, seesperaría quelas remuneracionesal trabajo en estas actividades fueran tal es que permitan condiciones decorosas en el bienestar dela población local. La evidencia muestra lo contrario.

CUADRO 1. Composición y costo diario dela canasta deconsumo observado por grupo alimenticio y producto. Piedras Negras, Coahuila, 1997.

\begin{tabular}{|c|c|c|c|c|}
\hline Grupo alimenticio & Productos & $\begin{array}{l}\text { Consumo por } \\
\text { hogar en } \\
\text { UAE (kg./ día) }\end{array}$ & $\begin{array}{l}\text { Precio promedio } \\
\text { por unidad } \\
\text { demedida }\end{array}$ & $\begin{array}{c}\text { Costo de } \\
\text { canastaen } \\
\text { pesos }\end{array}$ \\
\hline \multirow[t]{3}{*}{ Cereales } & Tortillademaíz & 0.763 & 2.500 & 1.908 \\
\hline & Pastas para sopa & 0.078 & 9.620 & 0.751 \\
\hline & Arrozengrano & 0.108 & 7.330 & 0.794 \\
\hline \multirow[t]{4}{*}{$\begin{array}{l}\text { Carnesy } \\
\text { procesados }\end{array}$} & $\begin{array}{l}\text { Carnederes(pulpa, } \\
\text { trozoy molida) }\end{array}$ & 0.087 & 25.870 & 2.253 \\
\hline & Carnedepollo & 0.460 & 9.529 & 4.384 \\
\hline & Jamón & 0.065 & 19.580 & 1.272 \\
\hline & Salchicha & 0.055 & 15.590 & 0.854 \\
\hline Lechey derivados & Lechepasterizada & 0.273 & 4.973 & 1.356 \\
\hline Huevo & Huevo & 0.187 & 12.703 & 2.376 \\
\hline Aceitesy grasas & A ceitevegetal & 0.103 & 8.986 & 0.924 \\
\hline Tubérculosy raíces & Papa & 0.270 & 3.821 & 1.030 \\
\hline \multirow[t]{4}{*}{ Verduras } & Tomaterojo & 0.152 & 9.094 & 1.383 \\
\hline & $\begin{array}{l}\text { Chileserranoy/ o } \\
\text { jalapeño }\end{array}$ & 0.071 & 6.253 & 0.442 \\
\hline & Cebolla & 0.111 & 5.245 & 0.585 \\
\hline & Lechuga & 0.049 & 6.877 & 0.340 \\
\hline Leguminosas & Frijol & 0.270 & 8.349 & 2.251 \\
\hline \multirow[t]{2}{*}{ Frutas } & Limón & 0.095 & 4.161 & 0.394 \\
\hline & Plátano & 0.141 & 5.579 & 0.787 \\
\hline Azucares & Azúcar & 0.209 & 6.533 & 1.366 \\
\hline \multirow[t]{3}{*}{ Bebidas } & Aguapurificada & 3.686 & 0.440 & 1.622 \\
\hline & $\begin{array}{l}\text { Refrescosy bebidas } \\
\text { gaseosas }\end{array}$ & 1.095 & 3.560 & 3.897 \\
\hline & Costototal & & & 30.966 \\
\hline
\end{tabular}

FUENTE: Elaboración propia con base en la encuesta sobre “Distribución del ingreso y patrones de consumo de la población del norte deCoahuila. La ciudad de Piedras Negras". El Colef, Piedras Negras, Coahuila 1997. 


\section{LA FRONTERA NORTE DE COAHUILA}

Por años, la población fronteriza del estado de Coahuila ha pugnado por una reclasificación dentro de las zonas salarial es que existen en el país desde 1986. Los municipios fronterizos del estado (Ocampo, Acuña, Jiménez, Piedras N egras, Nava, Guerrero eHidalgo) son de los pocos a nivel frontera norte que no están considerados en la zona salarial $A$ (de vida cara). ${ }^{5}$ La existencia de los puentes internacionales en las ciudades de Acuña y Piedras N egras, las hace establecer una relación de comercio importante con las ciudades vecinas, situación que ha determinado e impreso características de complementariedad para la población quelas habita, particularmenteen aspectos como el ingreso y consumo. Esta zona no ha sido ajena a desajustes de la economía nacional -como procesos devaluatorios- y sí, en cambio, han sido notorias las pérdidas del salario real frenteal costo económico devivir en tales ciudades.

El cuadro 2 muestra las diferencias regionales que existían en la determinación del monto salarial desdeaños antes dela conformación de la CNSM y la zonificación en áreas geográficas. Queda claro quelas regiones económicas con mayor impulso económico mantenían niveles salariales superiores al promedio estatal, eincluso al nacional, tal es el caso de Monclova, espacio minero e industrial preponderante por la ubicación de Altos Hornos de México (AHMSA), frontera norte puerta de entrada al mercado de ambos países, y la región de la Laguna, principal zona agrícola y ganadera del estado y del norte de México. Sin embargo, a partir de la década de los ochenta las diferencias regionales sefueron acortando hasta desaparecer, y el SM se mantuvo por abajo del promedio nacional (véase figuras 1y 2).

\footnotetext{
${ }^{5}$ Dentro dela zona salarial A seencuentran todos los municipios del estado deBaja California; los municipios deGuadalupe, Ciudad Juárez, Praxedis G. y Guerrero en Chihuahua; en Sonora: A gua Prieta, Cananea, N aco, N ogales, Plutarco Elías Calles, Puerto Peñasco, San Luis Río Colorado y Santa Cruz; mientras que en Tamaulipas se ubican todos los municipios fronterizos, más San Fernando.
} 
CUADRO 2. Salario mínimo diario nominal general, por región salarial en el estado de Coahuila, 1934-1986 (en pesos corrientes decada año).

\begin{tabular}{|c|c|c|c|c|c|c|c|}
\hline Periodo & $\begin{array}{c}\text { Prom. } \\
\text { nacional }\end{array}$ & $\begin{array}{c}\text { Prom.del } \\
\text { estado }\end{array}$ & Saltillo & Monclova & Norte & $\begin{array}{l}\text { Comarca } \\
\text { Lagunera }\end{array}$ & Oeste \\
\hline 1934-1935 & & 1.38 & & & & & \\
\hline 1936-1937 & & 1.43 & & & & & \\
\hline 1938-1939 & & 1.96 & & & & & \\
\hline 1940-1941 & & 1.99 & & & & & \\
\hline $1942-1943$ & & 1.60 & & & & & \\
\hline $1944-1945$ & & 2.12 & & & & & \\
\hline 1946-1947 & & 3.29 & & & & & \\
\hline 1948-1949 & & 3.69 & & & & & \\
\hline 1950-1951 & & 4.39 & & & & & \\
\hline 1952-1953 & & 5.28 & & & & & \\
\hline $1954-1955$ & & 7.47 & & & & & \\
\hline 1956-1957 & & 8.66 & & & & & \\
\hline 1958-1959 & & 11.20 & & & & & \\
\hline 1960-1961 & & 12.00 & & & & & \\
\hline 1962-1963 & & 12.00 & & & & & \\
\hline $1964-1965$ & 17.78 & 17.29 & 18.58 & 19.49 & 19.00 & 16.75 & 13.50 \\
\hline 1966-1967 & 20.90 & 19.79 & 19.40 & 23.09 & 22.15 & 19.50 & 15.50 \\
\hline 1968-1969 & 24.15 & 22.79 & 22.31 & 26.49 & 26.00 & 22.50 & 18.00 \\
\hline $1970-1971$ & 27.93 & 26.60 & 25.72 & 29.99 & 29.80 & 26.75 & 21.00 \\
\hline 1972 & 33.23 & 31.54 & 30.42 & 35.08 & 35.20 & 31.60 & 25.00 \\
\hline 1973 ( $1^{\circ}$ ene. $\left.-16 \mathrm{sep}\right)$ & 33.23 & 31.52 & 30.42 & 35.08 & 35.20 & 31.60 & 25.00 \\
\hline 1973 (17 sep.-31 dic.) & 39.20 & 37.20 & 35.90 & 41.38 & 41.55 & 37.30 & 29.50 \\
\hline 1974 ( $1^{\circ}$ ene. -7 oct.) & 45.03 & 42.96 & 40.43 & 47.23 & 47.90 & 44.40 & 33.00 \\
\hline 1974 (8 oct.-31 dic.) & 55.24 & 52.42 & 49.33 & 57.57 & 58.40 & 54.20 & 40.30 \\
\hline 1975 & 55.24 & 52.42 & 49.33 & 57.57 & 58.40 & 54.20 & 40.30 \\
\hline 1976 ( $1^{\circ}$ ene.-30 sep.) & 67.26 & 63.28 & 60.14 & 70.07 & 70.30 & 65.00 & 48.40 \\
\hline 1976 ( $1^{\circ}$ oct.-31 dic.) & 82.74 & 77.85 & 73.95 & 86.16 & 86.50 & 80.00 & 59.50 \\
\hline 1977 & 91.20 & 85.74 & 81.35 & 94.76 & 95.20 & 88.00 & 65.50 \\
\hline 1978 & 103.49 & 97.11 & 91.42 & 106.96 & 107.00 & 100.00 & 75.00 \\
\hline 1979 & 119.78 & 112.03 & 106.42 & 121.96 & 122.00 & 115.00 & 90.00 \\
\hline 1980 & 140.69 & 131.45 & 126.42 & 139.96 & 140.00 & 135.00 & 110.00 \\
\hline 1981 & 183.05 & 166.83 & 166.42 & 169.99 & 170.00 & 170.00 & 150.00 \\
\hline
\end{tabular}


CUADRO 2. Continuación.

\begin{tabular}{|c|c|c|c|c|c|c|c|}
\hline Periodo & $\begin{array}{c}\text { Prom. } \\
\text { nacional }\end{array}$ & $\begin{array}{c}\text { Prom. del } \\
\text { estado }\end{array}$ & Saltillo & Monclova & Norte & $\begin{array}{l}\text { Comarca } \\
\text { Lagunera }\end{array}$ & Oeste \\
\hline 1982 ( $1^{\circ}$ ene. -31 oct. $)$ & 244.83 & 221.09 & 220.53 & 225.00 & 225.00 & 225.00 & 200.00 \\
\hline 1982 ( $1^{\circ}$ nov. -31 dic. $)$ & 318.28 & 287.42 & 286.69 & 292.50 & 292.50 & 292.50 & 260.00 \\
\hline 1983 ( $1^{\circ}$ ene.-13 jun.) & 398.09 & 359.16 & 357.85 & 365.00 & 365.00 & 365.00 & 325.00 \\
\hline 1983 (14 jun.-31 dic.) & 459.01 & 415.06 & 413.67 & 421.00 & 421.00 & 421.00 & 380.00 \\
\hline 1984 ( $1^{\circ}$ ene. -10 jun. $)$ & 598.66 & & 54 & 00 & 550.00 & 550.00 & 495.00 \\
\hline 1984 (11 jun. -31 dic.) & 719.02 & 649.18 & 645.45 & 660.00 & 660.00 & 660.00 & 600.00 \\
\hline 1985 ( $1^{\circ}$ ene. -3 jun. $)$ & 938.81 & 851.60 & 860.00 & 860.00 & 860.00 & 860.00 & 780.00 \\
\hline 1985 (4 jun.-31 dic.) & $1,107.64$ & & $1,015.00$ & $1,015.00$ & $1,015.00$ & $1,015.00$ & 921.00 \\
\hline 1986 ( $1^{\circ}$ ene. -31 may $)$ & & & $1,340.00$ & $1,340.00$ & $1,340.00$ & $1,340.001$ & $1,340.00$ \\
\hline 1986 ( $1^{\circ}$ jun. -21 oct. $)$ & & & $1,675.00$ & $1,675.00$ & $1,675.00$ & $1,675.001$ & $1,675.00$ \\
\hline 1986 (22 oct.-31 dic.) & & & $2,060.00$ & $2,060.00$ & $2,060.00$ & $2,060.002$ & $2,060.00$ \\
\hline Zona salarial C & & & $2,535.00$ & $2,535.00$ & $2,535.00$ & $2,535.002$ & $2,535.00$ \\
\hline
\end{tabular}

FuENTE: CNSM, tomado de Estadísticas H istóricas de M éxico, tomo I, pp. 208-218.

La Junta Local de Conciliación y Arbitraje del Estado, no dictó la resolución correspondiente para el periodo 1934-1963, en que regían los salarios mínimos municipales establecidos por las Comisiones Especiales delos Salarios Mínimos; se presenta el promedio aritmético de dichos salarios mínimos municipales en cada entidad federativa. A partir de1964, en queinició sus funciones la CNSM, sepresentan los salarios mínimos generales establecidos para cada zona económica salarial, así como los promedios ponderados de dichos salarios por entidad federativa. Como ponderador se utilizó a la población asalariada para cada año, con base en estimaciones intercensales. En razón de los cambios que ha tenido la integración y la nomenclatura de las zonas económicas salariales en los últimos 20 años transcurridos a partir de 1964, se presenta la zonificación en uso a partir del 1 de enero de 1965, y cuando una zona ha sido el producto de la fusión de dos o más zonas, o de la integración de municipios a una zona, se presenta el promedio ponderado de los salarios mínimos vigentes en las zonas o municipios fusionados. Para conocer la integración de las zonas económicas salariales consúltese la publicación dela cNSM.

En contrapartida, el comportamiento del costo de vida fue en aumento; en este sentido, el mismo cuadro 2 nos muestra el salario mínimo nominal delazona C, a precios corrientes, queen comparación con el Índice Nacional de Precios al Consumidor (INPC) para tres ciudades del estado, refleja la variabilidad del salario real. 
ANÁLISISECONÓMICO DEL COSTO DE VIDA

Figura 1 y 2 
Es interesante observar que en el periodo comprendido entre 1995 a febrero de 1999, el INPC aumentó $116.46 \%$, mientras queel monto del salario en términos nominales ha sido menos variante; dehecho, para el mismo periodo sólo se otorgaron siete incrementos en el salario mínimo: $87.7 \%$ en términos acumulados. Por otra parte, el comportamiento del índicenos dicequela inflación promedio mensual es mayor en Ciudad de Acuña, particularmente desde el último mes del año 1995, seguida deM onclovay Torreón; es decir, el costo devidaesmayor para esta ciudad (véase las figuras 3 y 4).

El indicador generalmente utilizado para referirse a la capacidad de compra de la población es el salario real; en este sentido, si las variaciones mayores en precios sehan presentado en Acuña, y en vista de que la totalidad de los municipios del estado se ubican en la zona salarial $C$, es de esperarse que el salario mínimo real sea menor para esta ciudad; de hecho, esta ciudad se ha caracterizado por ser una de las más caras del país; si comparamos la inflación mensual deA cuña y el promedio general nacional, lo podemos observar, tal y como lo muestra la figura 3.

Un ejercicio que nos aproxima al anál isis de la capacidad de acceso a los elementos que componen la canasta de consumo observado construida para Piedras N egras, es comparar su costo con el número de perceptores de ingreso necesarios para adquirirla. La canasta está integrada por los bienes al imenticios que, por su frecuencia decompra, y por lo tanto consumo, es posi bleidentificar como consumo propio de la población local. En ella no seconsideran gastos devivienda, salud e higiene personal y educación. Su costo mensual para 1997 fuede 30.96 pesos corrientes, por lo queera necesario percibir 1.38salarios mínimos nominales diarios para acceder a ella.

Si evaluamos el ingreso percibido por la población ocupada en Piedras N egras, entre 1989 y 1997 vemos cómo en las actividades del sector industrial es donde existe un mayor número de trabajadores con percepciones de ingreso menores a dos salarios mínimos. No estamos diciendo quee pago a su fuerza detrabajo se real ice en estos términos, pues de hecho las negociaciones contractual es se pactan en niveles superiores a los aumentos dados a aquél, sin embargo, sí representa un tope en los acuerdos logrados. Y en consecuencia, los 
ANÁlisisECONÓmico del COSTO de VIDA

Figuras 3 y 4 
beneficiosson ínfimos. Así pues, si tomamos como medida dereferencia el SMD dela zonac, sorprendecómo una proporción importante-más de $50 \%$ en promedio- dela población ocupada seubica en estos rangos de ingreso (véase las figuras 5 y6).

A nteesta situación, como la hacen evidente Cortés y Rubal cava en su análisis sobredistribución del ingreso en México entre 1977y 1989, los cambios que se dieron en este periodo expresan las medidas de política económica tomadas como estrategia del model o dedesarrollo, pero, además, reflejan las estrategias que siguieron las familias en defensa desu capacidad deconsumo. En el caso particular dela frontera de Coahuila, la inserción al mercado de trabajo de un mayor número demiembros del hogar posibilita el acceso delasfamilias a los elementos deconsumo alimenticio diario, eigualmentea otra seriedesatisfactores. Sin embargo, las remuneraciones a la fuerza de trabajo por sí mismas no son suficientes para garantizar una reproducción física y social integral dela población.

Figura 5 
CUADRO 3: Salario mínimo nominal y real para ciudades del estado de Coahuila (zona “C”). Pesos de 1994. (julio 1994=100).

\begin{tabular}{|c|c|c|c|c|}
\hline Periodo & $\begin{array}{l}\text { Salario mínimo } \\
\text { diario nominal }\end{array}$ & Torreón & Mondova & Acuña \\
\hline 1995| 01 & 13.79 & 12.644 & 12.941 & 12.660 \\
\hline $1995 \mid 02$ & 13.79 & 12.273 & 12.317 & 12.169 \\
\hline 1995| 03/ 31 & 13.79 & 11.672 & 11.562 & 11.440 \\
\hline 1995| 04 & 15.44 & 11.981 & 12.039 & 11.958 \\
\hline 1995| 05 & 15.44 & 11.507 & 11.535 & 11.461 \\
\hline 1995| 06 & 15.44 & 11.259 & 11.148 & 11.078 \\
\hline 1995| 07 & 15.44 & 11.075 & 10.920 & 10.810 \\
\hline 1995| 08 & 15.44 & 10.868 & 10.717 & 10.606 \\
\hline $1995 \mid 09$ & 15.44 & 10.654 & 10.455 & 10.390 \\
\hline $1995 \mid 10$ & 15.44 & 10.456 & 10.147 & 10.104 \\
\hline 1995| 11 & 15.44 & 10.193 & 9.903 & 9.828 \\
\hline 1995| 12/ 03 & 17.00 & 10.876 & 10.520 & 10.459 \\
\hline 1996| 01 & 17.00 & 10.526 & 10.177 & 10.070 \\
\hline $1996 \mid 02$ & 17.00 & 10.302 & 9.947 & 9.799 \\
\hline 1996| 03 & 19.05 & 11.324 & 10.882 & 10.705 \\
\hline 1996| 04/ 01 & 19.05 & 11.058 & 10.641 & 10.436 \\
\hline 1996| 05 & 19.05 & 10.893 & 10.440 & 10.239 \\
\hline $1996 \mid 06$ & 19.05 & 10.736 & 10.272 & 10.060 \\
\hline 1996| 07 & 19.05 & 10.589 & 10.114 & 9.890 \\
\hline 1996| 08 & 19.05 & 10.448 & 9.978 & 9.753 \\
\hline $1996 \mid 09$ & 19.05 & 10.294 & 9.824 & 9.580 \\
\hline 1996| 10 & 19.05 & 10.152 & 9.578 & 9.368 \\
\hline $1996 \mid 11$ & 19.05 & 9.970 & 9.441 & 9.237 \\
\hline $1996 \mid 12 / 03$ & 22.5 & 11.505 & 10.911 & 10.651 \\
\hline 1997| 01 & 22.5 & 11.243 & 10.665 & 10.382 \\
\hline 1997| 02 & 22.5 & 11.066 & 10.492 & 10.206 \\
\hline 1997| 03 & 22.5 & 10.875 & 10.367 & 10.081 \\
\hline
\end{tabular}

Fuente: Cálculos propios con base en el cuadro dos y datos de salario e índices nacionales de precios al consumidor (Banco de México). El salario real es igual al salario nominal deflactado por el INPC por ciudad, para el estado de Coahuila. 
Figura 6

COMENTARIO FINAL

Con estos datos pretendemos dejar claro que los cambios habidos en la política salarial han mermado la capacidad de gran parte de la población deacceder a los el ementos necesarios desatisfacción básica, que esta situación se agrava más considerando las diferencias en el costo económico devivir en determinadas ciudades, concretamenteen ciudades fronterizas donde, tomando en cuenta que los patrones de consumo de la población pueden estar influenciados por los hábitos de las sociedades del vecino país del norte, ello vuelve más grave la situación para quienes viven en estas ciudades.

Finalmente, es importante decir que bajo esta nueva estrategia de desarrollo en el ámbito de los salarios, dada la pérdida de relación de éste con el costo de vida de la población y sus necesidades básicas, el logro de los objetivos de equilibrio macroeconómico a través de la contención salarial se ha logrado, sin embargo, esta estrategia no ha traído otro resultado más que la mayor pérdida de bienestar de la población. 
Deesta manera, esta situación es común para ciudades como Piedras Negras y Ciudad A cuña, a la que no se le ha reconocido su calidad fronteriza y donde, por continuar catal ogada en la zona salarial más baja del país, los aumentos a los SM y las negociaciones contractuales tienen como referencia el nivel mínimo de la zona c. Esta última situación condiciona el nivel salarial delos trabajadoressindicalizados, por ejemplo, los cuales han tratado de nivelar sus salarios a través de acuerdos contractuales con base en salarios profesionales (Quintero C.,1996,164); más allá de eso, el costo de vida resulta tan caro como en cual quier otra ciudad fronteriza al norte del país. ${ }^{6}$

Para los tomadores de las decisiones económicas en nuestro país, una política salarial basada en el costo de vida es válida sólo bajo la idea de que a un nivel mayor de salarios corresponda siempre una mayor producción, deotro modo, el incremento del poder adquisitivo terminarápor hacer subir los precios, y el desequilibrio generado puede disminuir la capacidad de competencia del país en los mercados internacionales. Sin embargo, los esfuerzos por aumentar la capacidad productiva de los trabajadores sefundamenta en el nivel educativo de la población, mismos que no se lograrán aumentar si la distribución funcional del ingreso, es decir la distribución entre trabajo y capital, tiende a concentrarse cada vez más.

La contracción salarial quepor años hemos resentido evidencia que la recuperación no será posible mientras no se deje de considerar al salario como freno inflacionario para lograr la estabilidad macroeconómica que el país requiere para mostrarse atractivo en el mercado internacional.

${ }^{6}$ En opinión dela autora, uno de los aspectos en que se muestra la difícil etapa por la que atraviesa el sindicalismo nigropetense es su incapacidad deflexibilizar aún más su política salarial. Véase Cirila Quintero Ramírez (1996), “Los sindicatos en la frontera coahuilense", en M emorias del II Simposium de estudios fronterizos, en el 146 aniversario de la ciudad de Piedras N egras, Coahuila, compilador Salvador Corrales, El Colegio dela Frontera N orte, R. Ayuntamiento del Municipio, ITPN. 


\section{BIBLIOGRAFÍA}

A boites Jaime (1995). "Sector informal, pobreza y flexibilidad laboral", en: Las nuevas políticas de ajuste en A mérica Latina, tomo I: Globalización del capital, reestructuración productivay empleo; PierreSalama, Federico Curiel, Juan Manuel Durán, JoséLorenzo Santos (comp.), editado por la Universidad de Guadalajara y el Centre D'Etudes Mexicaines et Centroaméricaines (CEMCA). México.

Boltvinik, Kalinka Julio (1987). "Ciudadanos de la pobreza y la marginación", en: revista El cotidiano, núm. 19, septiembrediciembre, UAM, México, D.F.

(1995). P obreza y estratificación social en M éxico, tomo X, Instituto Nacional de Estadística, Geografía e Informática, México D.F., mayo, pp. 66.

Cortés, Fernando y Rosa María Rubalcava (1991). A utoexplotación forzada y equidad por empobrecimiento, Colección Jornadas, 120, El Colegio de México. México D. F. 186 pp.

De la Garza Toledo, Enrique (1995). “Estilos de desarrollo y nuevos patrones derelaciones laborales", en: Las nuevas políticas deajuste en A mérica Latina, tomo I: Globalización del capital, reestructuración productiva y empleo; Pierre Salama, Federico Curiel, Juan Manuel Durán, JoséLorenzo Santos (comp.), editado por la Universidad de Guadalajara y el Centre D'Etudes Mexicaines et Centroaméricaines (CEMCA), México.

Díaz, Loría Eduardo (1991). “La recuperación de los salarios reales en México en un entorno de reducción y estabilización inflacionarias", en: Revista Comercio Exterior, vol. 41, núm. 4, México, D.F., abril, pp. 375-386.

Marinakis, Andrés (1995). "Nuevas tendencias en las políticas salariales", en: Revista de la CEPAL, núm. 57, Santiago de Chile, Chile, Comisión Económica para América Latina y el Caribe, diciembre, pp. 195.

Márquez Padilla, Carlos(1997). “La fijación delossalariosy el problema del empleo en los países de la OCDE. Una reflexión desde la perspectiva de México", en: El economista mexicano, vol. I, núm. 2, 
México D.F., Revista de El Colegio Nacional de Economistas, enero-marzo, pp. 121-144.

Oficinalnternacional del Trabajo (1983). Los salarios. M anual deeducación obrera, Ginebra, Suiza, Editorial Alfaomega, impreso en México, 1992.

Ordoñez Barba, Gerardo M. (1997). “Política social y letra muerta”, en: N exos, México, D.F., año 20, vol. XX, núm. 229, enero, pp. 22-24.

Ortega, Max (1995). "Congreso del Trabajo: crisis de sus funciones históricas y neocorporativismo"; en: Las nuevas políticas de ajuste en A mérica Latina, tomo I: Globalización del capital, reestructuración productiva y empleo, Pierre Salama, Federico Curiel, Juan Manuel Durán, J oséL orenzo Santos (comps.), editado por la Universidad deGuadalajaray el CentreD 'EtudesM exicaines et Centroaméricaines (CEMCA), Méx

ico.

Quintero, Cirila (1996). "Los sindicatos de la frontera coahuilense", en: Memorias del II Simposium deestudios fronterizos, Salvador Corrales (comp.). Piedras Negras, Coahuila, R. Ayuntamiento, 1995-1997, ITPN, El Colef.

Solís de Alba, A na Alicia (1995). “Neoliberalismo y flexibilización de las relaciones laborales en México (1982-1991); en: Las nuevas políticas de ajuste en A mérica Latina, tomo I: Globalización del capital, reestructuración productiva y empleo, Pierre Salama, Federico Curiel, Juan Manuel Durán, José Lorenzo Santos (comps.), editado por la Universidad de Guadalajara y el Centre D ÉEtudes Mexicaines et Centroaméricaines (CEMCA), México. 